\title{
On the Semiclassical Approach of the Heisenberg Uncertainty Relation in the Strong Gravitational Field of Static Blackhole
}

\author{
Fima Ardianto Putra
}

\begin{abstract}
Heisenberg Uncertainty and Equivalence Principle are the fundamental aspect respectively in Quantum Mechanic and General Relativity. Combination of these principles can be stated in the expression of Heisenberg uncertainty relation near the strong gravitational field i.e. $\Delta p \Delta r \geq \frac{\hbar / 2}{1-\frac{2 G M /\langle r\rangle}{c^{2}}}$ and $\Delta E \Delta t \geq \frac{\hbar / 2}{1-\frac{2 G M /\langle r\rangle}{c^{2}}}$. While for the weak gravitational field, both relations revert to $\Delta p \Delta r \sim \hbar / 2$ and $\Delta E \Delta t \sim \hbar / 2$. It means that globally, uncertainty principle does not invariant. This work also shows local stationary observation between two nearby points along the radial direction of blackhole. The result shows that the lower point has larger uncertainty limit than that of the upper point, i.e. $\Delta p_{A} \Delta r_{A}=\Delta p_{g} \Delta r_{g}\left(1-\frac{2 g H}{c^{2}}+\ldots\right)$. Hence locally, uncertainty principle does not invariant also. Through Equivalence Principle, we can see that gravitation can affect Heisenberg Uncertainty relation. This gives the impact to our's viewpoint about quantum phenomena in the presence of gravitation.
\end{abstract}

Keywords: Heisenberg Uncertainty Principle; Equivalence Principle; gravitational field.

\section{Introduction}

In the study of Hawking radiation, the very strong gravitational field near the Schwarezschild radius can generate pair production [1]. Particle move out and antiparticle move in toward the Schwarezschild radius. We have known that the pair production based on quantum field theory is explained by Heisenberg uncertainty [2]. On the other hand, the space and time around massive object like blackhole are affected by gravitation. There has been establish result of Schwarezschild metric that gravitational time dilation and length contraction along the radial coordinate are depend on the ratio of mass with respect to the radius of the blackhole [2]. That reason is intriguing for us to ask about how is the Heisenberg uncertainty in the context of the gravitational time dilation and length contraction?.

On another hand, Weak Equivalence Principle guides us to the understanding that inersial force equivalent with gravitational one [3]. It also valid if two forces are taken in to account relativisticly for the test particle [4]. As the consequence, if an integration is worked

Correspondence: ardiantoputraf2@gmail.com

Department of Physics, Universitas Jenderal Soedirman, Purwokerto, Indonesia

Full list of author information is available at the end of the article ${ }^{*}$ Equal contributor to both of them along the radial distance $r$ in local region, we obtain potencial energy difference. Then based on the energy conservation, we obtain kinetic energy difference that yields the velocity of particle with respect to the blackhole. This velocity depends on the mass-radius ratio, like the factor which makes gravitational time dilation and length contraction. So, equivalence principle provides explanation that relativistic kinetic energy and momentum depend on the ratio $M / r$. The question is how the ratio $M / r$ affects the uncertainty of both kinetic energy and momentum such a way that this phenomenon appears in the Heisenberg uncertainty representation?.

The question about whether ratio $M / r$ can affect the uncertainty of time, position, energy, and momentum or not, will bring us to the more fundamental question i.e. Does the Heisenberg uncertainty relation invariant in the presence of gravitation?. If it does, so it will give the impact to our's viewpoint about quantum phenomena in the presence of gravitation.

Based on the paper [5], Kentosh and Mohageg analyzed GPS (Global Positioning System) test to determine the LPI (Local Position Invariance) of Planck constant along the radial distance of the earth. The result shows that the Planck constant is invarian in the such limit which is parameterized by $\beta_{h}<0.007$, with $\beta_{h}$ explain a violation of LPI. 
It indicates the existence of limited invariance zone of Planck constant. The value of this constant will change along radial direction according to relation $\hbar_{x} / \hbar_{o}=\left(1+\beta_{h} \Delta U / c^{2}\right)$. So it is very reasonable to be connected with Heisenberg uncertainty invariance in the gravitational field.

Some considerations above, I propose to reconcile between General Relativity and Quantum Mechanic through both equivalence and uncertainty principle. Because those principles are very fundamental. Based on my hypothesis, properly they are linked uniquely such a way that can be oriented to the invariance aspect of Heisenberg uncertainty relation itself. In this paper, I study how the invariance of Heisenberg uncertainty relation in the gravitational field through Equivalence Principle to look for Heisenberg uncertainty which is consistent with the theory of gravitation.

In this study, I use the semiclasiccal approach by viewing the uncertainty relation in de Broglie wave packet. It is so, because according to the Ehrenfest theorem, the average value of uncertainty connects with the classical domain. So, it can be viewed in the context of Einstein relativity theory.

\section{Equivalence Principle in Ehrenfest Theorem's Viewpoint}

Special Relativity is the theory which prevails in the flat spacetime, while General Relativity prevails in the curve one. So Special Relativity is just locally consistent with respect to General Relativity [2]. It can be said that in the tiny region of vaccum space around the massive object, the condition approximates Special Relativity (i.e. the tangen space) which Relativistic Quantum Mechanic i.e. superposition principle can be valid enough. It requires that $g^{\mu v} \approx \eta^{\mu v}$ or specificly $g^{\mu v}(p)=\eta^{\mu v}(p)$. This is what Equivalence Principle means. In this condition, locally we can build pseudo-Euclidean (Minkowskian) coordinate by transforming the metric tensor of general coordinate $g^{\mu v}$ to the metric tensor of Cartesian (inersial) coordinate $\eta^{\mu v}[2]$.

Equivalence principle shows that according to the non-inersial (accelerated) observers i.e. observers that are placed stationary in the tiny regions along radial distance from the massive object's surface up to the very far place which gravitation is weak, They can view that they are in the inersial reference frames (flat space) with presence of gravitation that is viewed as a type of force [6]. Because we cannot distinguish these frames with non-inersia (accelerated) reference frame where inersia force appears $[2,6]$, so $F_{i}=F_{g}$ i.e. $m_{0} a=\frac{G M m_{0}}{r^{2}}$ (in the form of non-relativistic). It shows that an object in the tiny region of vacuum space around the massive object, the gravitation field is almost uniform so it cannot be distinguished with the uniform accelerated frame $[2,3,4,6]$.

When we integrate both left and right hand side of $m_{0} a=\frac{G M m_{0}}{r^{2}}$ with respect to the radial distance, so based on the energy conservation we obtain the relation between $v$ and gravitational potential which has been established in Newtonian picture, i.e. $\frac{1}{2} m_{0} v^{2}=\frac{G M m_{0}}{r^{2}}$. This equation can be obtained from equivalence principle in the non-relativistic form which $v$ is the escape speed of particle from gravitational bound of a static object with mass $M$ [7], In the context of blackhole, if $v=c$, so $r=R_{S}$, but the energy is still in the non-relativistic form. Then by using $v^{2}=\frac{2 G M}{r}$, we obtain the relativistic kinetic energy of particle which is written in the following binomial expantion

$$
\frac{1}{2} m_{0} v^{2}+\frac{3}{8} \frac{m_{0} v^{4}}{c^{2}}+\ldots=\frac{G M m_{0}}{r}+\frac{3}{8} \frac{m_{0} G^{2} M^{2}}{c^{2} r^{2}}+\ldots
$$

The first term of both left and right hand side are like in Newtonian form with the addition terms giving the relativistic correction. If Eq.(1) is expressed by Lorentz factor, we get that $\frac{m_{0} c^{2}}{\sqrt{1-\frac{v^{2}}{c^{2}}}}-m_{0} c^{2}=$ $\frac{m_{0} c^{2}}{\sqrt{1-\frac{2 G M}{r c^{2}}}}-m_{0} c^{2}$. It means that in the relativistic form, equivalence principle prevails, such as in the blackhole case [4]. From the relation above, we can state that $\gamma=1 / \sqrt{1-\frac{2 G M / r}{c^{2}}}$. It shows that the particle velocity is the vector field (the function of $r$ ) around the blackhole. It is the consequence of equivalence principle. Based on the Ehrenfest theorem, $v$ is connected with average velocity $\langle v\rangle$ i.e. the group velocity $v_{g}$ in the picture of de Broglie wave packet that exhibits correspondence to the classical limit $[8,9]$.

Before we discuss about $\langle v\rangle$, we will explain about acceleration previously. Based on Ehrenfest theorem, acceleration is written in the non-relativistic form as $\frac{d^{2}\langle r\rangle}{d t^{2}}=\frac{\left\langle-\frac{d V(r)}{d r}\right\rangle}{m_{0}}=\frac{\langle F(r)\rangle}{m_{0}}=-\frac{G M}{\left\langle r^{2}\right\rangle}$ [10], with $\left\langle r^{2}\right\rangle=\left\langle\Delta r^{2}\right\rangle$. So $-\frac{G M}{\left\langle r^{2}\right\rangle}$ is not equal to $-\frac{G M}{\left\langle r^{2}\right\rangle}$ as the classical form. It means that $\langle F(r)\rangle=F(\langle r\rangle)+$ $(r-\langle r\rangle) F^{\prime}(\langle r\rangle)+\ldots$. This equation to be $\langle F(r)\rangle \cong$ $F(\langle r\rangle)$ if $(r-\langle r\rangle) \cong 0$. In this condition, the change of potential is slowly with respect to the distance $[8,9,10]$ like in the local laboratory where equivalence principle prevails. We know that $F^{\prime}(\langle r\rangle)$ and the higher order do not give physical meaning in the context of Newton's second law because there is no term $a^{\prime}(\langle r\rangle)$ moreover in higher order, although in the form of arbitrary force equation itself such as gravitational force gives the meaning. The existence of $a^{\prime}(\langle r\rangle)$ does 
not obey equivalent principle because it is not uniform acceleration. So we must view that $F^{\prime}(\langle r\rangle)=0$. Then because of relativistic case, we take $(r-\langle r\rangle) \cong \lambda_{C}$, with $\lambda_{C}$ is the Compton wavelength as the smallest possible uncertainty $[11,12]$. Automatically it yields $\left\langle r^{2}\right\rangle \neq\langle r\rangle^{2}$. So $\langle F(r)\rangle \neq F(\langle r\rangle)$. In this condition $\langle a(r)\rangle=a_{1}$ and $a(\langle r\rangle)=a_{2}$, where $a_{1}$ and $a_{2}$ are the different constant such a way that acceleration does not depend on radial distance in the local laboratory. Then, integration of $\frac{d^{2}\langle r\rangle}{d t^{2}}$ gives $\langle v\rangle=\left\langle v_{0}\right\rangle+a_{1} t$ and the square form yields $\langle v\rangle^{2}=\left\langle v_{0}\right\rangle^{2}+2 a_{1}\left(\left\langle v_{0}\right\rangle+\frac{1}{2} a_{1} t^{2}\right)$. Suppose that $\left\langle v_{0}\right\rangle=0$, such a way that we get the relation between $\langle v\rangle$ and $\langle r\rangle$ as follow:

$$
\langle v\rangle^{2}=2 \frac{G M}{\left\langle r^{2}\right\rangle}\langle r\rangle \approx 2 \frac{G M}{\langle r\rangle^{2}}\langle r\rangle=\frac{2 G M}{r}
$$

\section{Heisenberg Uncertainty in the Gravitational Field}

The energy is possed by particle in the gravitational field is consist of relativistic kinetic and potential energy, but we will only take relativistic kinetic energy part instead of potential one in order to get uncertainty which is just contributed by kinetic energy. In special relativity, kinetic energy is $K=E-m_{0} c^{4}$. Then, here we use dispersion theory to view the uncertainty $[8,10,13]$, so $\Delta E=\frac{p}{E} c^{2} \Delta p=\frac{1}{v_{p h}} c^{2} \Delta p=v_{g} \Delta p$. This uncertainty relation can be described naturally by de Broglie wave packet $[8,10]$. It means that superposition principle can be used here but for local observation only where does Equivalence Principle prevails. Based on the $K=E-m_{0} c^{2}$, we get that $\Delta E=\Delta K$.

If the stationary observer in such event $P$ measures the test particle which is freely falling near the blackhole, so according to this observer, energy and momentum uncertainty of the test particle are $\Delta E=\gamma^{3} m_{0} v_{g} \Delta v$ and $\Delta p=\gamma^{3} m_{0} \Delta v$, with $\Delta v=$ $\sqrt{\left\langle v^{2}\right\rangle-\langle v\rangle^{2}}$ following standard deviation rule. Then here we define $\Delta K_{v}=v_{g} \Delta p_{v}$. As the consequence of Eq. (2), we just replace group velocity inside the Lorentz factor as follow:

$$
\begin{aligned}
& \Delta E=\frac{\Delta K_{v}}{\left(1-\frac{2 G M /\langle r\rangle}{c^{2}}\right)^{3 / 2}} \\
& \Delta p=\frac{\Delta p_{v}}{\left(1-\frac{2 G M /\langle r\rangle}{c^{2}}\right)^{3 / 2}}
\end{aligned}
$$

We can write $\Delta K_{v}=m_{0} v_{g} \Delta v \approx m_{0} \mid \frac{-G M}{\langle r\rangle^{2} \mid} \Delta t$ as the consequence of Eq. (2). It gives understanding as if energy and momentum respectively is the function of position and time such that contradiction with Heisenberg uncertainty itself. We can understand this by the following explanation.

Let's notice again Eq.(2). If we take uncertainty of this equation we get that $v_{g} \Delta v \approx \mid \frac{-G M}{\langle r\rangle^{2} \mid \Delta r}$ with $\sqrt{\left\langle v^{2}\right\rangle-\langle v\rangle^{2}}=\sqrt{\left\langle\Delta v^{2}\right\rangle}$ and $\sqrt{\left\langle r^{2}\right\rangle-\langle r\rangle^{2}=\sqrt{\left\langle\Delta r^{2}\right\rangle}}$. The maximum fixed value of $\left\langle v^{2}\right\rangle=c$, but $\langle v\rangle$ can change from zero at infinity distance up to $c$ at the horizon. While, the maximum fixed value of $\left\langle r^{2}\right\rangle=\infty$ up to Schwareszchild radius $r_{S}$. When the particle rests at infinity distance of blackhole, we can set $\Delta v \approx 0$ and $\Delta r \approx \infty$ correspond to the condition in which $\langle v\rangle \approx 0$ and $\langle r\rangle \approx \infty$. This condition is suitable with $v_{g} \Delta v \approx\left|\frac{-G M}{\langle r\rangle^{2}}\right| \Delta r$. Then if the particle closes to the horizon, we also take $\Delta v \approx 0$ and $\Delta v \approx \infty$ correspond to the condition that $\langle v\rangle \approx c$ and $\langle r\rangle \approx r_{S}$. So it gives that $v_{g} \Delta v \ll\left|\frac{-G M}{\langle r\rangle^{2}}\right| \Delta r$. So it gives that $v_{g} \Delta v \approx$ $\left|\frac{-G M}{\langle r\rangle^{2}}\right| \Delta r$. It will not be consistent with equivalence principle in the classical correspondence. The problem is how to keep the uncertainty form of Eq. (2) is still consistent for $\Delta v \approx 0$ and $\Delta r \approx \infty$. It is impossible. The way out/of this problem is viewing Lorentz Fitz-Gerald contraction effect to position uncertainty $\Delta r$. This effect will be explained evidently in the next section after we formulate position uncertainty in Eq.(5). Because of Lorentz Fitz-Gerald contraction to $\Delta r$, it shows that $\Delta r \approx 0$ not $\Delta r \approx \infty$ at a point close to horizon. In this condition, of course that $\langle a(r)\rangle \approx a(\langle r\rangle)$. Hence the uncertainty form of Eq.(2) is valid in all condition. But we must take the consequence that Heisenberg uncertainty seems to be violated because of $\Delta r \approx 0$ for $\Delta v \approx 0$. It is no problem because it is just position uncertainty which is according to the observation. The position uncertainty which will be infinite is the proper position uncertainty i.e. $\Delta r_{p}$ (see Eq.(5)), such that does not commute with velocity and not violates Heisenberg uncertainty principle. This point will has been clear later, in the last formulation of relativistic Heisenberg uncertainty. Then from relation $v_{g} \Delta v \approx\left|\frac{-G M}{\langle r \mid\rangle^{2}}\right| \Delta r$, we can cancel $v_{g}$ so that $\Delta v \approx\left|\frac{-G M}{\langle r \mid\rangle^{2}}\right| \Delta t$. When $\Delta v \approx c$ and $\Delta r \approx \lambda_{C}$ correspond to the condition that $\langle v\rangle \approx 0$ and $\langle r\rangle \approx \infty$, so $\Delta t \approx 0$ following Lorentz-Fitzgerald contraction of $\Delta r$.

Further from the $g^{\mu v} \approx \eta^{\mu v}$, locally we can define Cartesian coordinate system as follow [2] $d s^{2} \approx$ $c^{2} d t^{2}-d r^{2}-r^{2} d \theta^{2}-r^{2} \sin ^{2} \theta d \varphi^{2}$, but we will write in the sense of uncertainty i.e. $\Delta s^{2}=c^{2} \Delta t^{2}-\Delta r^{2}-$ $r^{2} \Delta \theta^{2}-r^{2} \sin ^{2} \theta \Delta \varphi^{2}$. It is good enough in describing local flat space time with $\Delta \theta=\Delta \varphi=0$, because particle just radially moves. The $\Delta r$ can be stated as $\Delta r=v_{g} \Delta t$. It is the contracted width of wave packet 
during the time $\Delta t$. So, we obtain uncertainty relation between position $r$ and time $t$ which can form the relation $\Delta E \Delta t=\Delta p \Delta r$. So there is analogy between energy-time and momentum-position uncertainty.

In quantum field theory, $r$ and $t$ are treated as a parameter, hence both of them are not properties of particle $[14,15]$. The $\Delta t$ and $\Delta r$ respectively show how long and how far the state changes correspond to the arbitrary observable $\mathrm{Q}[8,16]$. These process are describe as $\Delta t=\frac{1}{\frac{d Q}{d t}} \Delta Q$ and $\Delta r=\frac{v_{g}}{\frac{d Q}{d t}} \Delta Q$. Nevertheless, in the context of Heisenberg uncertainty, we can view both $\Delta r$ and $\Delta t$ as if an uncertainty such a way that can be connected with $\Delta p$ and $\Delta E$.

In Special Relativities viewpoint, we know that position-time uncertainty $(\Delta r-\Delta t)$ according to the stationary observer is expressed as $\Delta r=\gamma^{-1} \Delta t_{p}$. Then, equivalence principle shows that

$$
\begin{aligned}
& \Delta r=\Delta r_{p} \sqrt{1-\frac{2 G M /\langle r\rangle}{c^{2}}} \\
& \Delta t=\Delta t_{p} \sqrt{1-\frac{2 G M /\langle r\rangle}{c^{2}}}
\end{aligned}
$$

with $\Delta r_{p}$ are the proper length but $\Delta t_{p}$ is not the proper time duration. I state like that because the truly definition of proper time is $\Delta \tau=\Delta x_{p} / c$, not $\Delta t_{p}=\Delta x_{p} / v_{g}$. Then because of treating $\Delta r$ as the length, it is oriented to Lorentz Fitz-Gerald contraction and by cancelling $v_{g}$, the time is shorter also. So $\Delta r$ as the width of wave packet of freely falling particle when it is moving with $v_{g}$ close to the speed of light and $\Delta t$ as the duration for the moving wave packet to spend it's width (not time dilation), which are measured by the stationary observer. If we want to view that the time runs slower, we have to use the Lagrangian in expressing the energy, consistent with the rule that $\int L d t=-\sqrt{1-\frac{v^{2}}{c^{2}}} m_{0} c^{2} \tau / \sqrt{1-\frac{v^{2}}{c^{2}}}$. Hence $\Delta L=\gamma m_{0} v_{g} \Delta v=\frac{\Delta K_{v}}{\sqrt{1-\frac{2 G M /\langle r\rangle}{c^{2}}}}$ and $\Delta t=$ $\Delta t_{p}=\frac{\Delta t_{p}}{\sqrt{1-\frac{2 G M /\langle r\rangle}{c^{2}}}}$. It means that according to the stationary observer, the time is elapsed by the freely falling test particle near the blackhole runs slower. As the consequence, the spreading of the wave packet is slower also.

So we can state that Heisenberg uncertainty relation of the freely falling test particle according to the stationary observers in every local laboratory along the radial distance obey the relation

$$
\Delta p \Delta r=\frac{\hbar / 2}{1-\frac{2 G M /\langle r\rangle}{c^{2}}}
$$

$$
\Delta E \Delta t=\frac{\hbar / 2}{1-\frac{2 G M /\langle r\rangle}{c^{2}}}
$$

Then, from the Lagrangian form and time dilation, we get $\Delta L \Delta t=\frac{\hbar / 2}{1-\frac{2 G M /\langle r\rangle}{c^{2}}}$. Equation (7) and (8) are analogue each other but not for Lagrangian-time dilation form. So we will not use this form later. If we notice, the right hand side of Eq.(7) and (8) to be the minimum limit of Heisenberg uncertainty in relativistic domain with $\hbar / 2=\Delta p_{v} \Delta r_{p}$ for Eq. (7) and $\hbar / 2=\Delta K_{v} \Delta t_{p}$ for Eq. (8). We can give the terminology that the left hand side of Eq.(7) until Eq.(8) as the coordinate Heisenberg uncertainty while the right hand side, i.e. $\Delta p_{v} \Delta r_{p}$ and $\Delta K_{v} \Delta t_{p}$ whose value are $\hbar / 2$ are the proper Heisenberg uncertainty. It is like the concept of proper length/time and coordinate length/time. It means that globally, Heisenberg uncertainty minimum limit does not invariant. We can state that $\Delta K_{v}, \Delta p_{v}$, $\Delta t_{p}$, and $\Delta r_{p}$ are the forms which correspond to the non-relativistic uncertainty. Those forms can be understood as uncertainty of particle if the particle is far away from gravitational influence such a way that $\Delta p \Delta r \sim \Delta p_{v} \Delta r_{p}$ and $\Delta E \Delta t \sim \Delta K_{v} \Delta t_{p}$. So, the weak gravitational field will be not significant to increase the Lorentz factor in the uncertainty relation of particle. Equation (7) and (8) are consistent in the each tangen space where special theory of relativity prevails, i.e. the tiny region of space along the radial vacuum space around the blackhole.

Notice that $\Delta K_{v}$ according to freely falling observer S' is not equal to $\Delta K_{v}$ according to stationary observer $\mathrm{S}$ which correspond to the relation $\Delta E=\gamma^{3} \Delta K_{v}$, because in this relation, $\Delta K_{v}$ contains $v_{g}$ whose value close to $c$, while $v_{g}$ which is convenient to freely falling observer is 0 , so it seems contradictive that $\left(\Delta K_{v}\right)_{S}>\left(\Delta K_{v}\right)_{S^{\prime}}$. As the consequence $\left(\Delta t_{p}\right)_{S}<$ $\left(\Delta t_{p}\right)_{S^{\prime}}$ (because of relation $\left.\hbar / 2=\Delta K_{v} \Delta t_{p}\right)$. It shows the difference duration of $\Delta t_{p}$ between which is observed by S and S'. Nevertheless it is no problem, because $\left(\Delta K_{v} \Delta t_{p}\right)_{S}=\left(\Delta K_{v} \Delta t_{p}\right)_{S^{\prime}}=\hbar / 2$. Hence we can still take $\Delta K_{v} \Delta t_{p}$ according to stationary $\mathrm{S}$ observer in to account.

\section{Local Observation between Nearby Points}

A local laboratory where is equivalence principle prevails, requires that gravitational field must be uniform. Suppose that stationary observer has a local laboratory that it's height is $H$, connecting the upper point A and the lower point B. Freely 
falling particle moves from A to B as it is observed by two stationary observers. The form of relativistic momentum uncertainty at $\mathrm{A}$ is $(\Delta p)_{A}$ and at $\mathrm{B}$ is $(\Delta p)_{B}$. Then the relativistic form of energy uncertainty at $\mathrm{A}$ is $(\Delta E)_{A}$ and at $\mathrm{B}$ is $(\Delta E)_{B}$. According to two local stationary observers at $\mathrm{A}$ and $\mathrm{B}$, they relatively see relativistic energy-momentum uncertainty increases when the particle is freely falling from A to B. The observers them self who stay at two points would feel the difference of their energy-momentum uncertainty if we view they as a particle which are identic with the freely falling particle. While for the non-relativistic momentum uncertainty $\left(\Delta p_{v}\right)_{A}>\left(\Delta p_{v}\right)_{B}$ because $v_{g}$ at $\mathrm{B}$ is greater than that at $\mathrm{A}$, while the $\left\langle v^{2}\right\rangle=c$. So we can insert multiplication factor $\xi$ for $\Delta p_{v}$ between $\mathrm{A}$ and $\mathrm{B}$ to be $\left(\Delta p_{v}\right)_{A}=\xi\left(\Delta p_{v}\right)_{B}$, and so $\left(\Delta p \gamma^{-3}\right)_{A}=\xi\left(\Delta p \gamma^{-3}\right)_{B}$. Then for non-relativistic energy uncertainty at $\mathrm{A}$ is $\left(\Delta K_{v}=v_{g} \Delta p_{v}\right)_{A}$ and at B is $\left(\Delta K_{v}=v_{g} \Delta p_{v}\right)_{B}$. We can see that when $v_{g}$ at $\mathrm{A}$ is smaller, so the $\Delta p_{v}$ is greater. It is conversely at $\mathrm{B}$. Hence we get that the $\xi$ itself is $v_{g B} / v_{g A}$. Consequently it yields $\left(\Delta K_{v}\right)_{A}=\left(\Delta K_{v}\right)_{B}$, and so $\left(\Delta E \gamma^{-3}\right)_{A} \equiv \xi$ $\left(\Delta E \gamma^{-3}\right)_{B}$. Then, by using binomial expansion for the Lorentz factor, we obtain

$$
\begin{aligned}
& \Delta p_{A}=\xi \Delta p_{B}\left(1-\frac{3 g H}{c^{2}}+\ldots\right) \\
& \Delta E_{A}=\xi \Delta E_{B}\left(1-\frac{3 g H}{c^{2}}+\ldots\right)
\end{aligned}
$$

Further, because of $\left(\Delta p_{v}\right)_{A}=\xi\left(\Delta p_{v}\right)_{B}$, it gives the consequence that $\left(\Delta r_{p}\right)_{A}$ will be not equal to $\left(\Delta r_{p}\right)_{B}$ and because of $\left(\Delta K_{v}\right)_{A}=\left(\Delta K_{v}\right)_{B}$, the $\left(\Delta t_{p}\right)_{A}$ will be equal to $\left(\Delta t_{p}\right)_{B}$. Hence $\left(\Delta r_{p}\right)_{A}=$ $\xi^{-1}\left(\Delta r_{p}\right)_{B}$, so $(\gamma \Delta r)_{A}=\xi^{-1}(\gamma \Delta t)_{B}$. The $\Delta r-\Delta t$ is the space-time uncertainty of freely falling particle according to observer at two points and $\Delta r_{p}-\Delta t_{p}$ is the non-relativistic form of spacetime uncertainty . By using the same procedure i.e. binomial expansion, we get

$$
\begin{aligned}
& \Delta r_{A}=\xi^{-1} \Delta r_{B}\left(1+\frac{g H}{c^{2}}+\ldots\right) \\
& \Delta t_{A}=\Delta t_{B}\left(1+\frac{g H}{c^{2}}+\ldots\right)
\end{aligned}
$$

It shows that in the local laboratory, the Stationary observer will relatively see the difference of width and moving time of wave packet between upper point $\mathrm{A}$ and lower point B. Wave packet at B is shorter than that at A like in Lorentz-Fitzgerald contraction according to stationary observer and also for it's elapsing time. Stationary observers also feel that their radial length contracts.

Lorentz contraction happen globally along radial distance correspond to Eq.(5). Every wave packet shows local observation region where gravitation is uniform. If we zoom in this region, the width of wave packet changes with respect to the change of radial velocity correspond to Eq.(11) because of uniform gravitational field $g$. It means that locally, the state of particle is changed by $g$. This condition is shown in Figure 1.

The change of the width of wave packet is not caused by localizing process, but that is the Lorentz contraction effect. In this case, we do not localize particle.

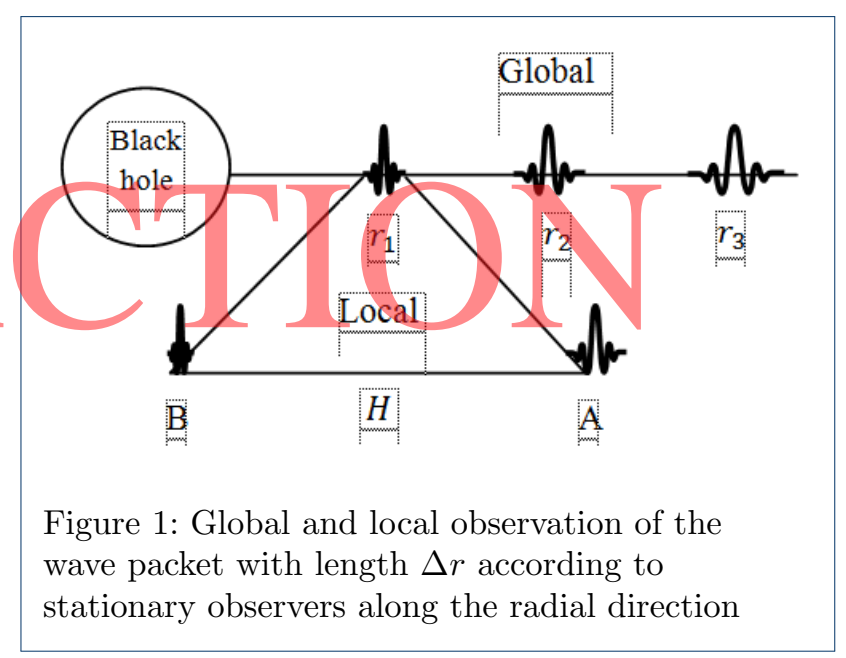

Then, in every local laboratory, the difference of Heisenberg uncertainty between two nearby point A and $\mathrm{B}$ are

$\Delta p_{A} \Delta r_{A}=\Delta p_{B} \Delta r_{B}\left(1-\frac{3 G g H}{c^{2}}+\ldots\right)\left(1+\frac{g H}{c^{2}}+\ldots\right)$

with uncertainty terms follow Eq.(7) and (8). It means that even locally, Heisenberg uncertainty minimum limit does not invariant also (Compare this with Eq.(7) and (8) which show globally not invariant). Equation (13) tells that in the local zone Stationary observer B see relatively that Heisenberg uncertainty of freely falling particle at him is larger than that at observer $\mathrm{A}$. It is because $\left(\Delta p_{v} \Delta r_{p}\right)_{B}$ is viewed as $(\Delta p \Delta r)_{B}$ at the lower point, while $\left(\Delta p_{v} \Delta r_{p}\right)_{A}$ is viewed as $(\Delta p \Delta r)_{A}$ at the upper one whose value is smaller than that at the lower. At the same moment, Freely falling particle will relatively see the Heisenberg uncertainty of the lower observer increases than that of the upper if the 
observers are viewed as identical particles like freely falling one. Observer B can feel that his uncertainty is larger than that of $\mathrm{A}$, because they are in the inersial frame with the presence of gravitational effect, while freely falling particle cannot feel that it's uncertainty increases, because it is in the inersial frame in the absence of gravitational effect.

According to paper [5], relation $\hbar_{x} / \hbar_{0}=$ $\left(1+\beta_{h} \Delta U / c^{2}\right)$ shows that the Planck constant varies depend on the potential difference which the contribution is determined by parameter $\beta_{h}$. If the Planck constant satisfies LPI (Local Position Invariance), then $\hbar_{x}$ would be constant. However, if $\hbar_{x}$ varies with position, then there is a concept of the change of invariance about Planck constant. This result theoretically can be understood based on the setting that $(\Delta p)_{A}=$ $\xi(\Delta p)_{B},(\Delta E)_{A}=(\Delta E)_{B},(\Delta r)_{A}=\xi^{-1}(\Delta r)_{B}$, $(\Delta t)_{A}=(\Delta t)_{B}$. So they can be formed to the several equations as follow:

$$
\begin{aligned}
\Delta p_{v A} & =\xi \Delta p v B\left(1+\frac{3 g H}{c^{2}}+\ldots\right) \\
\Delta K_{v A} & =\Delta K v B\left(1+\frac{3 g H}{c^{2}}+\ldots\right) \\
\Delta r_{p A} & =\xi^{-1} \Delta r p B\left(1-\frac{g H}{c^{2}}+\ldots\right) \\
\Delta t_{p A} & =\Delta t p B\left(1-\frac{g H}{c^{2}}+\ldots\right)
\end{aligned}
$$

This setting is equivalent with one we have done from Eq.(9) until Eq.(12). If-we use viewpoint of this setting for Local Observation between two nearby Point, so it means that we have taken $\hbar / 2$ for $\Delta p_{v} \Delta r_{p}$ and $\Delta K_{v} \Delta t_{p}$. Consequently, we can view that $\Delta p_{v} \Delta r_{p}$ and $\Delta K_{v} \Delta t_{p}$ are not as $\hbar / 2$ again. Both $\Delta p_{v} \Delta r_{p}$ and $\Delta K_{v} \Delta t_{p}$ can decrease even until zero at the horizon. Hence uncertainty relation between two stationary observers will become

$$
\left(\Delta p_{v} \Delta r_{p}\right)_{A}=\left(\Delta p_{v} \Delta r_{p}\right)_{B}\left(1+\frac{3 g H}{c^{2}}+\ldots\right)\left(1-\frac{g H}{c^{2}}+\ldots\right)
$$

with $\left(\Delta p_{v} \Delta r_{p}\right)_{A}=\hbar_{A}=\gamma-2 \Delta p_{A} \Delta r_{A}$ and $\left(\Delta p_{v} \Delta r_{p}\right)_{B}=\hbar_{B}=\gamma-2 \Delta p_{B} \Delta r_{B}$. Equation above can be used to give the reason from theoretical viewpoint in explaining the LPI violation of the Planck constant if we set that to the non- relativistic limit, because LPI violation data is analyzed for the weak gravitational field, i.e. the gravitation of earth. The analysis of LPI gives $\frac{\hbar_{A}}{\hbar_{B}}=\left(1+\beta_{h} \Delta U / c^{2}\right)$, while theoretically I get $\frac{\hbar_{A}}{\hbar_{B}}=\left(1+2 \Delta U / c^{2}\right)$ from Eq. (11).
Nevertheless, we must understand that although the set of Eq.(14) is equivalent with Eq.(9) until (12), but we cannot use this set. The reason is that when we use the set of Eq.(14), so the value of Eq (7) and (8) will be $\hbar / 2$ in the left hand side, while the $\hbar / 2$ in the right one will decrease up to zero at the horizon. It means that the minimum limit of Heisenberg uncertainty can than $\hbar / 2$. Whereas, commonly in quantum mechanics, the minimum limit of Heisenberg uncertainty must be $\hbar / 2$ as the proper value. So I decide to use the previous manner in the sense that Heisenberg uncertainty proper limit is $\hbar / 2$, and it will be larger in the strong gravitational field. In this condition, the states of the particle is constrained by larger phase space $p-r$ than that at a point which far away from the blackhole. At the horizon, Heisenberg uncertainty will be infinite. For the weak gravitational field, we get $\Delta p_{A} \Delta r_{A} / \Delta p_{B} \Delta r_{B}=\left(1-2 \Delta U / c^{2}\right)$. It is consistent with quantum mechanic minimum limit $\hbar / 2$ but contradict with $\hbar_{A} / \hbar_{B}=\left(1+2 \Delta U / c^{2}\right)$. It can be the subject for the next study.

Heisenberg uncertainty in this study is actually more appropriate for the real particles which fall to the blackhole, while particles in Hawking radiation are virtual. However we can use these equations also practically to describe vacuum fluctuation, because principally, that phenomenon connected with uncertainty. The relation between ratio $M / r$ and Heisenberg uncertainty in this study can unify universal constants $G, \hbar$, and $c$. The discussion which implicates the Planck scale and the concept of quantum gravity [17, 18, 19] was explained by generalized uncertainty relation $\Delta p \Delta x \geq \hbar / 2\left[1+\beta(\Delta p)^{2}+\ldots\right]$. This relation also shows the unification of $G, \hbar$, and $c$. The discussion about relation between that form and the result in this paper is beyond of this paper. Principally, both of those forms show that the minimum limit of Heisenberg uncertainty will increase even blowing up in a such situation.

\section{Conclusion}

Heisenberg uncertainty relation in the strong gravitational field is $\Delta p \Delta r \geq \frac{\hbar / 2}{\left(1-\frac{2 G M /\langle r\rangle}{c^{2}}\right)}$ and or $\Delta E \Delta t \geq \frac{\hbar / 2}{\left(1-\frac{2 G M /\langle r\rangle}{c^{2}}\right)}$. While the forms revert to $\Delta p \Delta x \sim \hbar / 2$ and or $\Delta E \Delta t \sim \hbar / 2$ if gravitational field is weak where is the non-Relativistic Quantum Mechanic prevails. For the local observation between two nearby points, the uncertainty is $\Delta p_{A} \Delta r_{A}=\Delta p_{B} \Delta r_{B}\left(1-\frac{2 g H}{c^{2}}+\ldots\right)$. Unification between general relativity and quantum domain based on my understanding gives the Heisenberg uncertainty relation as the function of gravitational 
field with expression of three fundamental constants. This uncertainty relation is the result from combining equivalence principle and uncertainty principle. Through equivalence principle, gravitation can affect Heisenberg uncertainty relation.

\section{Author}

\section{Fima Ardianto Putra}

Dari :

(1) Department of Physics, Universitas Jenderal Soedirman

\section{References}

1. Hawking SW. Black holes and thermodynamics. Physical Review D. 1976;13(2):191.

2. Hobson MP, Efstathiou GP, Lasenby AN. General relativity: an introduction for physicists. Cambridge University Press; 2006.

3. Carroll SM. Spacetime and geometry. Cambridge University Press; 2019.

4. Carmeli M. Classical Fields: General Relativity and Gauge Theory,(1982). John Wiley and Sons Inc;

5. Kentosh J, Mohageg M. Global positioning system test of the local position invariance of Planck's constant. Physical review letters. 2012;108(11):110801.
Cheng TP. Relativity, gravitation and cosmology: a basic introduction. Cheng TP. Relativity, gravitation and
vol. 11. Oxford University Press; 2009.

7. Ferraro R. Einstein's space-time: An introduction to special and general relativity. Springer Science \& Business Media; 2007.

8. Morrison MA. Understanding quantum physics: A user's manual. vol. 1. Addison-Wesley; 1990.

9. Gasiorowicz S. Quantum Physics, John Wiley\&Sons. University of Minnesota; 2003.

10. Eisberg R, Resnick R. Quantum Physics of Atoms, Molecules, Solids, Nuclei, and Particles John Wiley \& Sons. Inc; 1985.

11. Wachter A. Relativistic quantum mechanics. Springer Science \& Business Media; 2010.

12. Greiner W. Quantum mechanics: an introduction. Springer Science \& Business Media; 2011.

13. Greiner W. Relativistic Wave Equations, 3rd English edition. Springer-Verlag, Berlin/Heidelberg; 2000.

14. Ryder LH. Quantum field theory. Cambridge university press; 1996.

15. Mandl F, Shaw G. Quantum field theory. John Wiley \& Sons; 2010.

16. Aitchison IJR, Hey AJG. Gauge Theories in Particle Physics: A Practical Introduction: From Relativistic Quantum Mechanics to QED. vol. 1. CRC Press; 2012.

17. Kempf A, Mangano G. Minimal length uncertainty relation and ultraviolet regularization. Physical Review D. 1997;55(12):7909.

18. Kempf A, Mangano G, Mann RB. Hilbert space representation of the minimal length uncertainty relation. Physical Review D. 1995;52(2):1108.

19. Adler RJ. Six easy roads to the Planck scale. American Journal of Physics. 2010;78(9):925-932. 